\title{
MOLECULAR BASIS OF CD22 FUNCTION AND THERAPEUTIC TARGETING
}

June Ereño-Orbea ${ }^{1}$, Taylor Sicard ${ }^{1,2}$, Hong Cui ${ }^{1}$, Mohammad T. Mazhab-Jafari ${ }^{1}$, Samir Benlekbir ${ }^{1}$, Alba Guarné ${ }^{3}$, John L. Rubinstein ${ }^{1,2}$, Jean-Philippe Julien ${ }^{1,2^{*}}$

${ }^{1}$ The Hospital for Sick Children Research Institute, Toronto ON, M5G 0A4. ${ }^{2}$ University of Toronto, Toronto, ON, M5S 1A8. ${ }^{3}$ McMaster University, Hamilton, ON, L8S 4L8.

"Corresponding author

CD22 is a B cell surface receptor that is a member of the sialic acid-binding immunoglobulin-like lectin family (Siglec). CD22 maintains a baseline level of B-cell inhibition by creating a threshold of antigen binding that must be achieved for B-cell activation to proceed. CD22 is an attractive and validated target for B-cell depletion in cases of autoimmune diseases and B-cell-derived malignancies. Numerous therapeutic approaches in development harness B-cell inhibition through CD22 to induce tolerance or anergy, or to deplete dysregulated B cells through CD22 targeting by either small molecules, or antibody-drug conjugates.

Here we present the crystal structure of the three most $\mathrm{N}$-terminal domains of the extracellular portion of human CD22 in the absence and presence of a2-6 sialyllactose glycan at 2.1 and $2.2 \AA$ resolutions, respectively. The sialic acid binding pocket is located within the first domain of CD22 and is constrained by a unique $\beta$-hairpin that confers specificity for $\alpha 2-6$ glycosylated ligands. Electron microscopy and small-angle Xray scattering studies showed that the full length CD22 extracellular domain adopts a limited range of conformations, indicating low flexibility and an elongated, tilted rod structure. Our structural characterization provides insights into how CD22 might interact in cis to bind other CD22 sialylated glycoproteins on the surface of B cells to create dynamic inhibitory nanoclusters, and in trans to self-ligands at the immune synapse, resulting in sustained B-cell inhibition as a mechanism to prevent autoimmunity.

The localization of CD22 in nanoclusters and its extensive N-linked glycosylation likely impact how CD22 can be targeted therapeutically. Consequently, we next characterized the antigenic surface of CD22 recognized by two leading therapeutic antibodies in clinical trials: epratuzumab and pinatuzumab. Binding competition between epratuzumab and pinatuzumab revealed they recognize non-overlapping epitopes on CD22. Moreover, we found that epratuzumab binding to CD22 is glycan dependent. To delineate the epratuzumab epitope at high resolution, we solved the crystal structure of CD22 in complex with epratuzumab Fab at 3.1 $\AA$ resolution, and uncovered that CD22 glycan at position N231 is part of the antibody epitope.

Our studies provide molecular insights into the mechanisms that govern B-cell inhibition and valuable clues for the design and improvement of CD22 modulators, either based on sialic acid-containing ligands or next-generation antibody therapeutics. 